\title{
RELATIONS BETWEEN TRANSVERSE MOMENTUM DISTRIBUTIONS AND CHIRAL ODD GENERALIZED PARTON DISTRIBUTIONS
}

\author{
SIMONETTA LIUTI* \\ Department of Physics, University of Virginia, \\ 382 McCormick Rd., Charlottesville, VA 22904, USA \\ sl4y@virginia.edu \\ GARY R. GOLDSTEIN \\ Department of Physics and Astronomy, \\ Tufts University, Medford, MA 02155, USA \\ gary.goldstein@tufts.edu \\ J. OSVALDO GONZALEZ HERNANDEZ \\ Department of Physics, University of Virginia, \\ 382 McCormick Rd., Charlottesville, VA 22904, USA \\ jog4m@virginia.edu \\ KUNAL KATHURIA \\ Department of Physics, University of Virginia, \\ 382 McCormick Rd., Charlottesville, VA 22904, USA \\ kktf@virginia.edu
}

\begin{abstract}
We discuss the information on the spin structure of the proton that one can access by studying transverse momentum distributions, generalized parton distributions and their connection through generalized transverse momentum distributions. A "flexible" parametrization is proposed to extract this information from deeply virtual exclusive experiments.
\end{abstract}

Keywords: Generalized parton distributions; transverse momentum distributions; hadronic spin structure.

\section{Introduction}

The concept of Generalized Parton Distribution (GPD) opened new ways for exploring the full partonic structure of hadrons, including their momentum, spatial, and angular momentum distributions (see Ref. [1] for a review). By recognizing the relation of GPDs and Transverse Momentum Distributions (TMDs) to Wigner Distributions through Generalized Parton Correlation Functions (GPCFs) [2], it has 
been possible to relate different views of nucleon structure - from momentum space to coordinate space - and to connect them to measurements of the longitudinal and transverse components of partonic motion. Besides the chiral even GPDs, $H$, $E, \widetilde{H}$, and $\widetilde{E}$, the transversity/chiral odd/quark helicity-flip GPDs $[3], H_{T}, E_{T}$, $\widetilde{H}_{T}$, and $\widetilde{E}_{T}$, also play an important role in this context. It was recently pointed out that chiral-odd GPDs can be extracted from Deeply Virtual Meson Production (DVMP) experiments $[4,5,6]$. Data from Jefferson Lab Hall B indeed show the dominance of the chiral-odd GPDs contribution in deeply virtual $\pi^{\circ}$ electroproduction $[7,8]$. In this contribution we review some of these concepts, and we introduce new multi-parton correlations (Section 2). A complete discussion of the latter is in preparation and will be presented elsewhere. In Section 3 we discuss our parametrization for GPDs in both the chiral even and chiral odd sectors. In Section 4 we draw our conclusions.

\section{Relations Between TMDs and GPDs: Unraveling New Multiparton Correlations}

The phenomomenology of GPDs and TMDs has been extensively developed in the past few years. In a nutshell, TMDs can be extracted from Semi-Inclusive Deep Inelastic Experiments (SIDIS), provided the fragmentation function for the detected hadron can be disentangled; GPDs are extracted by de-convoluting the Compton form factors from deeply virtual exclusive experiments, namely DVMP and Deeply Virtual Compton Scattering (DVCS). At variance with the more studied problem of determining Parton Distribution Functions (PDFs) from inclusive deep inelastic scattering, for either TMDs or GPDs the extraction from data requires some extra step that introduces some "procedural", or model dependence. Even more importantly, the question has arisen of "what type of information" can one deduce from semi-inclusive and exclusive type data, since both GPCFs and Wigner Distributions contain redundant terms that need to be carefully sorted out $[9,10]$.

In what follows we briefly review formalism which is valid for deeply virtual exclusive processes including both Deeply Virtual Compton Scattering (DVCS), or Deeply Virtual Meson Production (DVMP) (Fig. 1). We consider the following factorized form of the helicity dependent amplitudes [11],

$$
f_{\Lambda_{\gamma}, \Lambda ; \Lambda_{\gamma}^{\prime}, \Lambda^{\prime}}=\sum_{\lambda, \lambda^{\prime}} g_{\lambda, \lambda^{\prime}}^{\Lambda_{\gamma}, \Lambda_{\gamma(M)}^{\prime}}\left(X, k_{T}, \zeta, t ; Q^{2}\right) \otimes A_{\Lambda^{\prime}, \lambda^{\prime} ; \Lambda, \lambda}\left(X, k_{T}, \zeta, t\right),
$$

where $\Lambda_{\gamma}, \Lambda$ refer to the initial (virtual) photon and proton helicities, $\left(\Lambda_{\gamma(M)}^{\prime}, \Lambda^{\prime}\right)$ to the final particles' ones, and $\lambda^{\prime}, \lambda$ to the struck quark helicities. The convolution integral is given by

$$
\otimes \rightarrow \int_{-\zeta+1}^{1} d X d^{2} k_{T}
$$

$g_{\lambda, \lambda^{\prime}}^{\Lambda_{\gamma}, \Lambda_{\gamma(M)}^{\prime}}$ describes the partonic subprocess $\gamma^{*}+q \rightarrow \gamma(M)+q$, whereas $A_{\Lambda^{\prime}, \lambda^{\prime} ; \Lambda, \lambda}$ is the quark-proton helicity amplitude. In DVCS, at leading twist, one writes the 


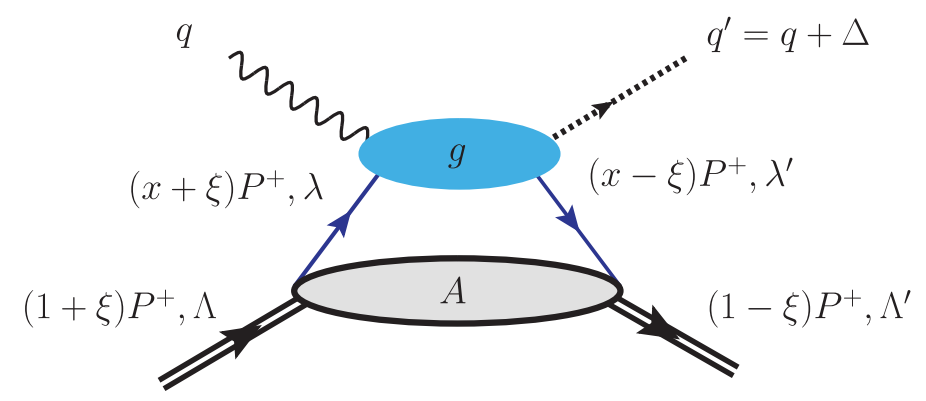

Fig. 1. Leading order amplitude for DVMP, $\gamma^{*}+P \rightarrow M+P^{\prime}$. For details on kinematics and notation see Ref. [1].

collinear factorized expression,

$$
\begin{aligned}
f_{+1, \Lambda ;+1 \Lambda^{\prime}}=\epsilon_{\mu}^{+1} T_{\Lambda \Lambda^{\prime}}^{\mu \nu} \epsilon_{\nu}^{*+1}= & \int_{-1+\zeta}^{1} d X\left[g_{++}^{1,1}+g_{++}^{-1,-1}\right]\left(A_{\Lambda^{\prime},+; \Lambda,+}+A_{\Lambda^{\prime},-; \Lambda,-}\right) \\
& +\left[g_{++}^{1,1}-g_{++}^{-1,-1}\right]\left(A_{\Lambda^{\prime},+; \Lambda,+}-A_{\Lambda^{\prime},-; \Lambda,-}\right)
\end{aligned}
$$

where

$$
\begin{gathered}
g_{++}^{1,1} \pm g_{++}^{-1,-1}=\sqrt{X(X-\zeta)}\left(\frac{1}{X-\zeta+i \epsilon} \pm \frac{1}{X-i \epsilon}\right)=\sqrt{X(X-\zeta)} C^{ \pm}, \\
A_{\Lambda^{\prime},+; \Lambda,+}+A_{\Lambda^{\prime},-; \Lambda,-}=\bar{U}\left(P^{\prime}, \Lambda^{\prime}\right)\left(\gamma^{+} H(X, \zeta, t)+\frac{i \sigma^{+\mu}\left(-\Delta_{\mu}\right)}{2 M} E(X, \zeta, t)\right) U(P, \Lambda) \\
A_{\Lambda^{\prime},+; \Lambda,+}-A_{\Lambda^{\prime},-; \Lambda,-}=\bar{U}\left(P^{\prime}, \Lambda^{\prime}\right)\left(\gamma^{+} \gamma_{5} \widetilde{H}(X, \zeta, t)+\gamma_{5} \frac{-\Delta^{+}}{2 M} \widetilde{E}(X, \zeta, t)\right) U(P, \Lambda)
\end{gathered}
$$

Similarly, the transversely polarized photon contribution to $\pi^{o}$ electroproduction reads [5],

$$
\begin{gathered}
f_{+1, \Lambda ; 0 \Lambda^{\prime}}=\epsilon_{T}^{\mu} T_{\mu}^{\Lambda \Lambda^{\prime}}=\int_{-1+\zeta}^{1} d X g_{+-}^{1,0} A_{\Lambda^{\prime},-; \Lambda,+}, \\
g_{+-}^{1,0}=g_{M}(Q) \sqrt{X(X-\zeta)}\left(\frac{1}{X-\zeta+i \epsilon} \pm \frac{1}{X-i \epsilon}\right)=g_{M}(Q) \sqrt{X(X-\zeta)} C^{ \pm}
\end{gathered}
$$

where $g_{M}(Q)$ describes the meson vertex, and

$$
\begin{aligned}
A_{\Lambda^{\prime},-; \Lambda,+}= & \bar{U}\left(P^{\prime}, \Lambda^{\prime}\right)\left(i \sigma^{+i} H_{T}^{q}(X, \zeta, t)+\frac{\gamma^{+} \Delta^{i}-\Delta^{+} \gamma^{i}}{2 M} E_{T}^{q}(X, \zeta, t)\right. \\
& \left.+\frac{\bar{P}^{+} \Delta^{i}-\Delta^{+} \bar{P}^{i}}{M^{2}} \widetilde{H}_{T}^{q}(X, \zeta, t)+\frac{\gamma^{+} \bar{P}^{i}-\bar{P}^{+} \gamma^{i}}{2 M} \widetilde{E}_{T}^{q}(X, \zeta, t)\right) U(P, \Lambda)
\end{aligned}
$$


Note that due to parity constraints, both in the chiral even and odd cases, out of the 8 quark-proton helicity amplitudes we construct 4 independent ones which in turn are written in terms of linear combinations of GPDs.

Sensible information is obtained when the GPDs are proportional to linear combinations of amplitudes that are diagonal in a given basis, meaning that the spin projections are the same on the LHS and RHS of the process (Fig. 1). This will allow us to associate each $k_{T}$ unintegrated GPD or GTMD, with a TMD carrying the same spin information. However, model independent relations between GPDs and TMDs cannot be established on a general basis, even once a connection between spin configurations is established, since GTMDs are amplitudes i.e. non diagonal objects in transverse momentum space (the quarks carry $k_{T}$ on the LHS and $k_{T}-\Delta_{T}$ on the RHS of Fig. 1), while TMDs are squares of amplitudes therefore diagonal by definition. The question about establishing systematics for connecting GPDs and TMDs was originally addressed in [12] and extended to the GTMD and GPCF substructure in [9]. Here we present a summary of recent work (in preparation): we write all GPDs in terms of quark-proton amplitudes for skewness, $\zeta=0$, in the Helicity, Canonical Transversity (spin along $y$-axis), Planar Transversity (spin along $x$-axis), and Mixed Bases (proton transverse spin, quark spin longitudinal), respectively. The structures we find connect with a similar analysis conducted for TMDs in [13], and with Refs.[12, 9].

\section{Chiral Even unintegrated GPDs:}

- $H\left(X, k_{T}, 0, t\right)$ is diagonal in all bases: helicity, canonical transversity, planar transversity, and mixed. For $t=0$ it connects to $f_{1}\left(x, k_{T}\right)$.

- $E\left(X, k_{T}, 0, t\right)$ is diagonal in the canonical transversity basis, i.e. it is an eigenstate of the nucleon transverse polarization.

- $\widetilde{H}\left(X, k_{T}, 0, t\right)$ is diagonal in helicity and non-diagonal in either transversity, i.e. it measures longitudinal polarization connecting for $t=0$ to $g_{1}\left(x, k_{T}\right)$.

- $\widetilde{E}\left(X, k_{T}, 0, t\right)$ is non-diagonal in helicity and transversity, and has a vanishing prefactor for $\zeta \Delta_{\perp} \rightarrow 0$. It is diagonal in the mixed longitudinal-transverse basis, i.e. it measures the longitudinal quark polarization in a transversely polarized nucleon, and it is connected to $g_{1 T}\left(x, k_{T}\right)$.

The Chiral Odd GPDs are non diagonal in the helicity basis, but they can be interpreted in terms of transversely polarized parton distributions.

\section{Chiral Odd unintegrated GPDs:}

- $H_{T}\left(X, k_{T}, 0, t\right)$ is diagonal in planar transversity.

- $H_{T}\left(X, k_{T}, 0, t\right)+\tau^{2} \widetilde{H}_{T}\left(\tau^{2}=\left(t_{0}-t\right) / 4 M^{2}\right)$, is diagonal in canonical transversity, and in planar transversity. It connects to transversity, $h_{1}\left(x, k_{T}\right)$ in the forward limit. - $\left[2 \widetilde{H}_{T}+E_{T}\right]\left(X, k_{T}, 0, t\right)$ is diagonal in canonical transversity.

- $\widetilde{H}_{T}\left(X, k_{T}, 0, t\right)$ is non-diagonal in either transversity bases. It becomes diagonal considering a mixed canonical and planar transversity representation. $\tau^{2} \widetilde{H}_{T}$, 
emerges as the difference between the canonical transversity non-flip amplitudes, and the planar transversity non-flip amplitudes. It is connected to $h_{1 T}^{\perp}\left(x, k_{T}\right)$.

- $\widetilde{E}_{T}\left(X, k_{T}, 0, t\right)$ vanishes at $\zeta=0$.

One can now classify GTMDs by the information they carry as either onebody or two-body density distributions. In other words, in this subsequent step we introduce an additional, complementary, physical criterion for interpreting the proton spin structure. For example, it has been postulated in Ref. [14] that

$$
f_{1 T}^{\perp} \leftrightarrow E
$$

whereas in Ref. [9] it was shown that $f_{1 T}^{\perp}=-F_{1,2}^{o}$ i.e. the imaginary part of the GTMD component with coefficient $i \sigma^{i+} k_{T}^{+}$, while $E=-F_{1,1}^{e}+2\left(k_{T} \cdot \Delta_{T} / \Delta_{T}^{2} F_{1,2}^{e}+\right.$ $\left.F_{1,3}^{e}\right)$, or a linear combination of the real components of the GTMD.

Here we point out another aspect that makes $E$ and $f_{1 T}^{\perp}$ intrinsically different despite their intuitive connection, in that we notice that the unintegrated GPDs correspond to one-body density distributions defined by

$$
\begin{aligned}
F(X, 0, t)= & \int d^{2} \mathbf{k}_{T} \int \frac{d z^{-}}{2 \pi} \frac{d^{2} \mathbf{z}_{T}}{(2 \pi)^{2}} \exp ^{i X P^{+}+z^{-} i \mathbf{k}_{T} \cdot \mathbf{z}_{T}} \\
& \times\left\langle P^{\prime}\left|\bar{\psi}\left(0, z^{-}, z_{T}\right) \Gamma^{+} \psi\left(0,0,0_{T}\right)\right| P\right\rangle \\
= & \sum_{\lambda} \int d^{2} \mathbf{k}_{T}\left\langle P^{\prime}\left|b^{\dagger}\left(k^{+}, k_{T}^{\prime} ; \lambda\right) b\left(k^{+}, k_{T} ; \lambda\right)\right| P\right\rangle \\
= & \int d^{2} \mathbf{k}_{T} \phi^{*}\left(X, \mathbf{k}_{T}-\mathbf{\Delta}\right) \phi\left(X, \mathbf{k}_{\mathbf{T}}\right) \\
= & \int d^{2} \mathbf{k}_{T} \tilde{\rho}_{1}\left(X, \mathbf{k}_{T}-\boldsymbol{\Delta}_{T} ; X, \mathbf{k}_{T}\right), \\
F(X, 0, t)= & \int d^{2} b e^{i \mathbf{b} \cdot \boldsymbol{\Delta}} \rho_{1}(X, \mathbf{b}),
\end{aligned}
$$

i.e. leading twist GPDs are defined by one-body density distributions that are respectively, diagonal in $X$, and non diagonal in transverse momentum as in Eq.(8a), and diagonal in both $X$ and impact parameter, b, as in Eq.(8b).

For an observable with Final State Interactions (FSI), e.g. the SSA related to $f_{1 T}^{\perp}[14]$, we find

$$
\begin{aligned}
& \left\langle k_{T}^{i}(x)\right\rangle_{U T} \\
& \quad=\int \frac{d z^{-}}{2 \pi} \exp ^{i X P^{+} z^{-}}\left\langle P\left|\bar{\psi}\left(0, z^{-}, 0_{T}\right) \Gamma^{+}\left[\mathcal{W}\left(z^{-}, 0\right) I_{q}\left(z^{-}\right)\right] \psi\left(0,0,0_{T}\right)\right| P\right\rangle \\
& \quad=\int d^{2} \mathbf{k}_{T} \int d^{2} \mathbf{l}_{T} \phi^{*}\left(X, \mathbf{k}_{T}\right) \phi\left(X, \mathbf{k}_{T}-\mathbf{l}_{T}\right) L\left(X, \mathbf{l}_{T}\right) \\
& \left\langle k_{T}^{i}(X)\right\rangle_{U T}=\int d^{2} \mathbf{b}_{1} d^{2} \mathbf{b}^{\prime}{ }_{1} d^{2} \mathbf{b}_{2} \rho_{2}\left[\left(X, \mathbf{b}_{1}\right),\left(0, \mathbf{b}_{2}\right) ;\left(X, \mathbf{b}^{\prime}{ }_{1}\right),\left(0, \mathbf{b}_{2}\right)\right] I\left(\mathbf{b}_{1}-\mathbf{b}_{2}\right),
\end{aligned}
$$


where $\rho_{2}$ is a semi-diagonal two-body density distribution. Eqs. (9a,9b) give "microscopic" information about the correlator/density distribution in the presence of FSI. The condition by which FSI factors out is that the intermediate states definining $\phi^{*}$ and $\phi$ in the correlator, are equal, namely $|X\rangle=\left|X^{\prime}\right\rangle$, in which case we obtain,

$$
\left\langle k_{T}^{i}(X)\right\rangle_{U T}=\int d^{2} \mathbf{k}_{T} \phi^{*}\left(X, \mathbf{k}_{T}\right) \phi\left(X, \mathbf{k}_{T}\right) L(X),
$$

in agreement with the phenomenological ansatz of Ref. [15]. In order to go beyond this simple approximation, one needs to consider the full two-body density in coordinate space displayed in Eq.(9b).

\section{Extracting GPDs from Experiment}

We now address the question of "how" to extract from experiment the new level of information that was outlined in the previous Section. GPDs are elusive objects to extract from experiment both because of the fact that they appear embedded in Compton form factors, and because they depend on two additional kinematical variables, which makes it much more challenging to construct a parametrization. While Neural Network base methods are been pursued $[16,17]$, here we present a user friendly version of our "flexible parametrization" for generalized parton distributions obtained from a recursive fitting procedure of available experimental data. The data included in our fit are from the flavor separated nucleon elastic form factors measurements, and from deep inelastic, and deeply virtual Compton scattering experiments. Additional information provided by lattice calculations of the higher moments of generalized parton distributions, is also considered. The parametrization's kinematical range of validity is in the region of Bjorken $0.1 \leq x \leq 0.85$, $1 \mathrm{GeV}^{2} \leq Q^{2} \leq 10 \mathrm{GeV}^{2}, 0 \leq-t \leq 1 \mathrm{GeV}^{2}$.

The parametrization is given in the following form,

$$
F(X, \zeta, t)= \begin{cases}F_{D G L A P}(X, \zeta, t) & \text { if } \zeta \leq X \leq 1 \\ F_{E R B L}(X, \zeta, t) & \text { if } 0<X<\zeta\end{cases}
$$

where $F \equiv H, E, \widetilde{H}^{\mathrm{a}}$

DGLAP region:

$$
F_{D G L A P}(X, \zeta, t)=G_{M_{X}, m}^{M_{\Lambda}}(X, \zeta, t) R_{p}^{\alpha, \alpha^{\prime}}(X, \zeta, t)
$$

$\underline{\text { ERBL region: }}$

$$
\begin{aligned}
& F_{E R B L}^{-}(X, \zeta, t)=a^{-}(\zeta, t) X^{2}-a^{-}(\zeta, t) \zeta X+H(\zeta, \zeta) \\
& F_{E R B L}^{+}(X, \zeta, t)=a^{+} X^{3}-a^{+} \zeta X^{2}+c X+d
\end{aligned}
$$

${ }^{a}$ The GPD $\widetilde{E}$ is different in that it has a non trivial contribution from the pion pole; we will discuss it in a separate paper. 

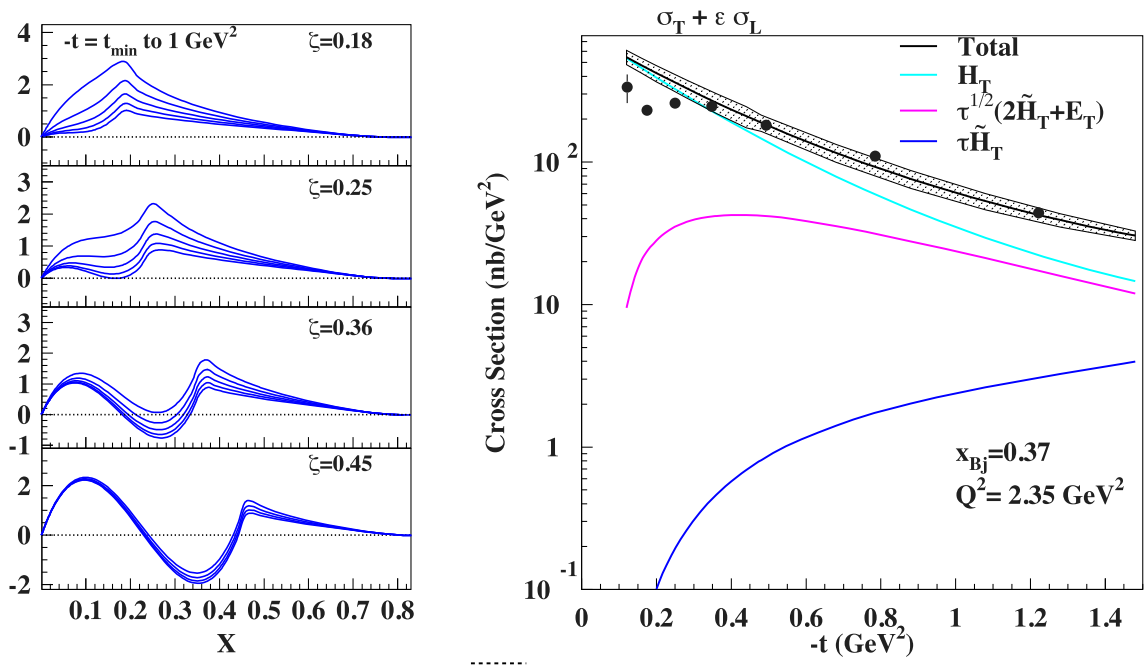

Fig. 2. Left: $H_{u}\left(X, \zeta, t ; Q^{2}\right)$ evaluated at $Q^{2}=2 \mathrm{GeV}^{2}$. Each panel shows $H_{u}$ plotted vs. $X$ at different values of $\zeta=0.18,0.25,0.36,0.45$, and $-t$ from $t_{\min }=-M^{2} \zeta^{2} /(1-\zeta)$ to $1 \mathrm{GeV}^{2}$ (adapted from Ref. [11]). Right: contribution of the various chiral odd GPDs to $d \sigma_{T} / d t+\epsilon_{L} d \sigma_{L} / d t$ from our model, plotted vs. $-t$ for $x_{B j}=0.37$, and $Q^{2}=2.35 \mathrm{GeV}^{2}$, along with data from [7].

where $F^{-}$and $F^{+}$follow from the crossing symmetry relations where in the nonsymmetric system of variables adopted here the axis of symmetry is shifted to $X=\zeta / 2$.

The functions $G_{M_{X}, m}^{M_{\Lambda}}$ and $R_{p}^{\alpha, \alpha^{\prime}}$ are respectively, the quark-diquark and Regge contributions from Ref. [11]. The diquark model was made more general by letting the mass of the diquark system vary according to a spectral distribution. Extending the diquark mass values generalization corresponds to "reggeizing" the covariant model since the spectral distribution can then reproduce the Regge behavior which is necessary to describe the low $X$ behavior. This scenario was first considered in Ref. [18], a similar model was more recently developed in Ref. [19], where the reggeization procedure was discussed at great length; in Ref. [20], using a similar perspective, Regge behavior was "implanted" in the Double Distributions. In our parametrization the Regge term is considered multiplicatively. By taking a spectral function $\rho_{R}\left(M_{X}^{2}\right) \propto\left(M_{X}^{q}\right)^{2\left[\alpha+\alpha^{\prime}(x) t-1\right]}$ one has,

$$
\begin{aligned}
F(X, \zeta, t) & =\mathcal{N} \int_{\bar{M}_{X}^{2}}^{\infty} d M_{X}^{2} M_{X}^{2\left[\alpha+\alpha^{\prime}(x) t-1\right]} G_{M_{X}, m}^{M_{\Lambda}}(x, 0, t) \\
& =\left.\mathcal{N} x^{-\left[\alpha+\alpha^{\prime}(x) t\right]} \int_{\bar{z}}^{\infty} d z z^{\left[\alpha+\alpha^{\prime}(x) t\right]} G_{M_{X}, m}^{M_{\Lambda}}(x, 0, t)\right|_{x=z / M_{X}^{2}} \\
& \approx \mathcal{N} x^{-\left[\alpha+\alpha^{\prime}(x) t\right]} G_{\bar{M}_{X}, m}^{M_{\Lambda}}(x, 0, t) .
\end{aligned}
$$

where the variable $M_{X}^{2}$ in $G_{M_{X}, m}^{M_{\Lambda}}$ has been replaced with $z=x M_{X}^{2}$ [21]. 
Our recursive procedure consists in first determining a few parameters which determine the $X$ dependence by fitting the chiral even GPDs to the PDFs. Keeping these parameters fixed, we then fit the nucleon form factors, and finally we use DVCS data to fit the additional $\zeta$ dependence. In Fig. $2 \mathrm{a}$ we show, for example, the resulting shape of the GPD $H$. With this parametrization in hand, we applied parity relations and crossing symmetries within the diquark model to obtain forms for the chiral odd GPDs. These enter the $\pi^{o}$ electroproduction cross section through Eqs. $(5,6,7)$. In Fig. 2b we show the different contributions of GPDs to the recent $\pi^{o}$ electroproduction data.

\section{Conclusions}

We addressed both questions of "what type of information" can one extract from deeply virtual exclusive experiments, and "how". The TMD-GPD connection via GTMDs and GPFCs plays a central role. In particular, we suggested that the SSA related quantities, $f_{1 T}^{\perp}$ and $h_{1}^{\perp}$ should be described by two-body density distributions, at variance with most model calculations. The effect of chiral-odd GPDs behavior from our model on pseudoscalar meson production was also shown.

This work was funded by U.S. D.O.E. grant DE-FG02-01ER4120.

\section{References}

1. A. V. Belitsky and A. V. Radyushkin, Phys. Rept. 418, 1 (2005).

2. A. V. Belitsky, X.-d. Ji and F. Yuan, Phys. Rev. D 69, 074014 (2004).

3. M. Diehl, Eur. Phys. Jour. C 19, 485 (2001).

4. S. Ahmad, G. R. Goldstein and S. Liuti, Phys. Rev. D79, 054014 (2009).

5. G. R. Goldstein, J. O. G. Hernandez and S. Liuti, arXiv:1201.6088 [hep-ph], to be published in J.Phys. G..

6. S. V. Goloskokov, P. Kroll, Eur. Phys. J. A47, 112 (2011).

7. V. Kubarovsky, I. Bedlinskiy et al. [CLAS Collaboration], Phys. Rev. Lett. 109, 112001 (2012).

8. E. Fuchey, A. Camsonne, C. Munoz Camacho, M. Mazouz, G. Gavalian, E. Kuchina, M. Amarian, K. A. Aniol et al., Phys. Rev. C 83, 025201 (2011).

9. S. Meissner, A. Metz and M. Schlegel, JHEP 0908, 056 (2009).

10. C. Lorce and B. Pasquini, Phys. Rev. D 84, 014015 (2011).

11. G. R. Goldstein, J. O. Hernandez and S. Liuti, Phys. Rev. D 84, 034007 (2011).

12. S. Meissner, A. Metz and K. Goeke, Phys. Rev. D 76, 034002 (2007).

13. M. Boglione and P. J. Mulders, Phys. Rev. D 60, 054007 (1999)

14. M. Burkardt, Phys. Rev. D 72, 094020 (2005).

15. A. Bacchetta and M. Radici, Phys. Rev. Lett. 107, 212001 (2011).

16. K. Kumericki, D. Muller and A. Schafer, JHEP 1107, 073 (2011).

17. S. Liuti, K. Holcomb and E. Askanazi, arXiv:1112.2280 [hep-ph].

18. S. J. Brodsky, F. E. Close and J. F. Gunion, Phys. Rev. D 8, 3678 (1973).

19. A. P. Szczepaniak, J. T. Londergan and F. J. Llanes-Estrada, Acta Phys. Polon. B 40, 2193 (2009).

20. A. V. Radyushkin, Phys. Rev. D 83, 076006 (2011).

21. J. O. Gonzalez-Hernandez, S. Liuti, G. R. Goldstein and K. Kathuria, arXiv:1206.1876 [hep-ph], submitted for publication in Phys. Lett. B. 